\title{
CMR in Pericardial Diseases - an Update
}

\section{Eleftherios Vidalakis ${ }^{1} \cdot$ Michalis Kolentinis $^{1} \cdot$ Monika Gawor $^{1} \cdot$ Moises Vasquez $^{1} \cdot$ Eike Nagel $^{1}$}

Published online: 3 March 2020

(C) The Author(s) 2020

\begin{abstract}
Purpose of Review To review the latest developments and the current role of the cardiac magnetic resonance (CMR) in pericardial diseases and their complications.

Recent Findings Cardiac Magnetic Resonance (CMR) has the ability to incorporate anatomy, physiology, and "virtual histology" strategies to achieve the most accurate diagnosis for even the most demanding, pericardial diseases.

Summary Acute, chronic, recurrent, and constrictive pericarditis as well as pericarditis related complications, pericardial masses and congenital pericardial defects are commonly encountered in clinical practice with relatively significant morbidity and mortality. Owing to the challenging diagnosis, CMR imaging is often employed in confirming the diagnosis and elucidating the underling pathophysiology. In this review we outline the common CMR techniques and their expected diagnostic outcomes.
\end{abstract}

Keywords Pericarditis · Pericardial effusion · Tamponade $\cdot$ Cardiovascular magnetic resonance $\cdot$ T1 and T2 mapping $\cdot$ Late gadolinium enhancement $\cdot$ Tagging $\cdot$ Real-time phase contrast $\cdot$ Cardiac masses

\section{Introduction}

The pericardium can be affected by inflammatory processes, infectious, iatrogenic, and malignant diseases, metabolic disorders as well as congenital disease. All the above can cause pericarditis, fluid accumulation, tamponade or constriction, as well as concomitant myocarditis [1]. Even though, over the last decades, the mortality related to pericardial disease has been constantly decreasing [2], morbidity has remained a considerable and unconquered problem [3]. The main challenge remains a prompt diagnosis and targeted management guided by the underlying pathophysiology and mechanisms as well as the activity of the disease, all of which requires an accurate diagnostic method which can be safely applied serially to allow monitoring of treatment $[4,5]$. According to current clinical practice, the diagnosis of the pericardial diseases, such as pericarditis, is mainly based on the symptoms, the ECG, and the echocardiography. Due to the fact that the above

This article is part of the Topical Collection on Cardiac Magnetic Resonance

Eike Nagel

eike.nagel@ cardiac-imaging.org

1 Institute for Experimental and Translational Cardiovascular Imaging, DZHK Centre for Cardiovascular Imaging, Goethe University Frankfurt, University Hospital Frankfurt am Main, Germany diagnostic techniques require considerable pericardial effusion in order to uncover any pathology of the pericardium, they lack of sensitivity especially in subclinical cases and those cases with no or minimal pericardial effusion that unfortunately represent the majority. Noninvasive imaging has a great armamentarium for accurate diagnosis and treatment guidance of pericardial diseases. In comparison to computed tomography (CT), single photon emission tomography (SPECT), and positron emission tomography (PET), cardiac magnetic resonance (CMR) has major advantages. It is a very powerful investigation tool which adds important information in order to elucidate this, often complex and multifaceted disease [6]. In this review we will summarize the classic CMR sequences that are used for this entity and review novel methods which utilize both contrast and non-contrast techniques in order to strengthen our diagnostic capacity.

\section{Current and Novel CMR Sequences}

1) Balanced Steady-State Free Precession (bSSFP) is the sequence which is most frequently used for cine CMR imaging. Owing to high spatial and temporal resolution, it provides information about ventricular interdependence, pericardial rigidity as well as the anatomy of the pericardium and the neighboring tissues [7]. bSSFP also 
has a T1/T2 signal providing considerable contrast between fluid and myocardium.

2) Mapping techniques like native T1 and T2 mapping sequences, are the most accurate way for tissue characterization. The generation of exact numbers and pixel-wise color maps allows for discrimination of normal myocardium, fibrous tissue, fat, and water, as well as uncovering concomitant myocardial inflammation superimposed to pericarditis [8, 9], (Fig. 1). Mapping techniques have already replaced many of the old-fashioned imaging approaches like the classic black-blood T1 weighted spinecho MR imaging as well as the T2 weighted spin-echo imaging, by providing more information about the anatomy and the histology of the pericardium and the neighboring tissues $[10,11]$.

3) Inversion recovery prepared $\mathrm{T} 1$ weighted imaging with intravenous gadolinium based contrast agent (GBCA) is a contrast-enhanced imaging technique that detects masses or inflammation of the pericardium as well as concomitant pathologies of the myocardium or neighboring tissues. Due to its strong contrast between normal tissue and regional fibrosis, late gadolinium enhancement imaging (LGE) is the most important classic technique for evaluating the pericardial diseases followed by early enhancement (EGE) and first pass perfusion imaging [12]. A novel approach reduces examination time, costs and contrast-agent exposure by using a fast low-dose GBCA rest protocol, such as with $0.1 \mathrm{mmol} / \mathrm{kg}$ of gadobutrol, with no significant loss of information [13]. Phase sensitive inversion recovery (PSIR) applied to LGE is widely used in clinical practice by improving the contrast between the normal and the inflamed pericardium. On PSIR reconstruction the fluid has negative amplitude (it is total black) rendering it easily distinguishable from any other tissue.

4) CMR myocardial tagging sequences and CMR real-time phase contrast (RT-PC) flow measurement technique are two techniques which add information about specific entities like constrictive pericarditis [14, 15].

\section{Normal Pericardium and CMR}

On CMR the normal pericardium is indistinguishable from pericardial fat unless thickened (more than $4 \mathrm{~mm}$ ). On SSFP cine CMR the normal pericardial fluid appears bright and slightly more intense than pericardial fat. The normal pericardium is only mildly enhanced by GBCA due to scarcity of vessels to deliver the contrast media, although this can change in case of an inflammatory process with neovascularization [6]. LGE with PSIR has the ability to depict even the smallest amount of pericardial fluid as a black area which contrasts to the white pericardium when present. By using this technique we are able to discriminate the fluid (black) from the fat (white). A more recent approach is the use of native T1 and T2 mapping techniques, which have replaced many of the classic approaches such as T1 weighted imaging and T2 weighted imaging by offering a precise tissue discrimination [8]. Fat has very low $\mathrm{T} 1$ times and appears as black in native T1 mapping. The above information can be added in PSIR-LGE imaging to differentiate the enhanced-inflamed pericardium from the normal pericardial fat (both are white in PSIR-LGE imaging).

\section{Pericarditis and CMR}

Pericarditis is an inflammatory pericardial syndrome with variable causes such as infections, autoimmune diseases, idiopathic, traumatic or iatrogenic damages, radiation or metabolic diseases. The clinical diagnosis of pericarditis is mostly based with two of the following criteria; (1) chest pain (>85-90\%), (2) pericardial friction rub ( $\leq 33 \%)$, (3) electrocardiogram (ECG) changes $(\leq 60 \%)$, and (4) pericardial effusion ( $\leq 60 \%$ ). Additional supporting findings include: (a) evidence of markers of inflammation and (b) evidence of pericardial inflammation by an image technique (CT, CMR) [5]. Given the unspecific presentation which often could also been assigned to myocarditis or coronary artery diseases, advanced imaging is frequently used in clinical practice to decide on the diagnosis. Although echocardiography is proposed as the first diagnostic technique in patients with symptoms suggestive of pericarditis, it is of doubtful help, as it cannot assess pericardium, but infers the presence of pericarditis indirectly based on the presence of pericardial effusion. More often than not pericarditis happens without or with only minimal effusion hence ECG and echocardiography approaches of diagnosing pericarditis should be considered with caution.

CMR is very effective in detecting pericarditis because inflamed pericardium is enhanced by GBCA. Pericardial LGE uptake for detection of pericardial inflammation has been reported to range from $94 \%$ to $100 \%$. The most common cause in developed countries is viral infections (80\% to $90 \%$ of cases), whereas in developing countries and HIV patients the leading cause is tuberculosis. Of all hospital admissions and chest pain admissions, $0.1 \%$ and $5 \%$ respectively are attributed to pericarditis [16]. Frequently pericarditis can be the first clinical expression of a malignancy [17]. A more subclinical course of pericarditis is often seen in patients with autoimmune diseases, like systemic lupus erythematosus (SLE), and which is accompanied by myocarditis [18]. LGE imaging is also an effective CMR technique to guide treatment of pericarditis [19]. The presence of pericardial LGE despite standard medical therapy in patients with ongoing symptoms should prompt the clinician to consider either a dose increase or prolongation of the duration of the therapy [20], (fig. 2). Further 
Fig. 1 The contribution of native T1 mapping techniques to separate the fat from fluid. Four chamber view, bSSFP imaging (a) and Native T1 mapping technique (b). Pericardial fat (a), pericardial fluid (b), epicardial fat (c)
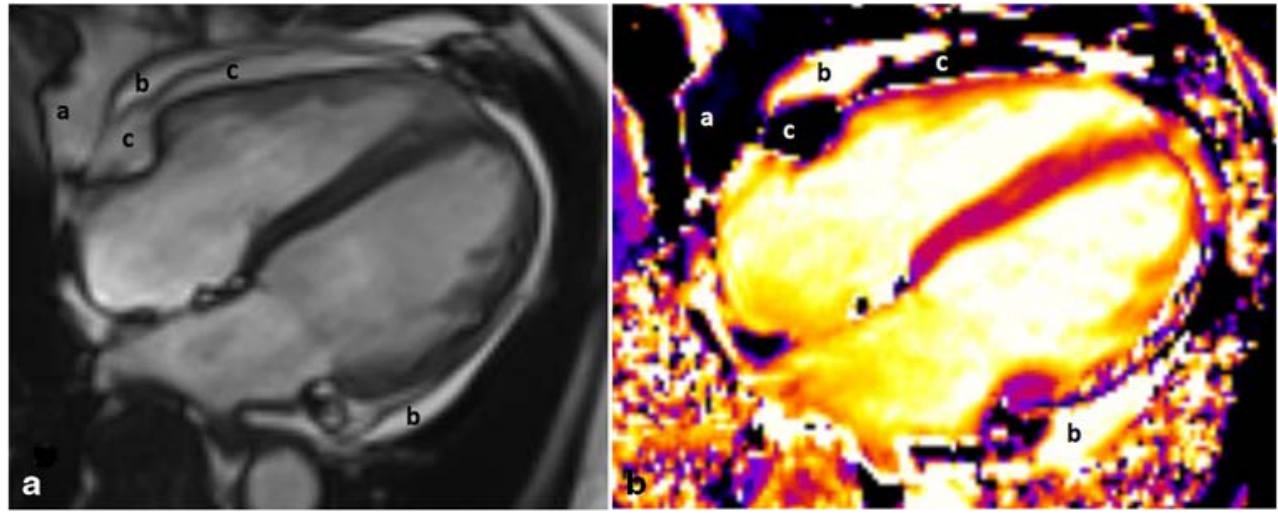

supportive signs are an increased thickness of the pericardium to more than $4 \mathrm{~mm}$ in cine bSSFP imaging and pericardial effusion, which is apparent in less than $40 \%$ of patients [21]. PSIR-LGE imaging is able to detect even minute amounts of fluid not visible by echocardiography [14].CMR is able to detect pericarditis in $>20 \%$ of patients with chest pain and exclusion of ischemia. The above further supports the notion of providing CMR to patients with chest pain but no ischemia to uncover the underlying pathophysiology of their chest pain [22]. Pericarditis often superimposed on myocarditis as they share the same causes like viruses and consequent auto- immune responses. This is of major importance, as these two entities need to be treated simultaneously. Myopericarditis seems to be more frequent among men with pericarditis (51\% vs 25\%) [23]. Detection of concomitant myocarditis is highly relevant for prognosis and treatment. Over the last decades, CMR is increasingly employed to address the question of myocardial involvement due to the above-mentioned ability for tissue characterization. While earlier reports have recommended a "2 out of 3" Lake Louise criteria approach for detecting myocardial inflammation, this approach has been challenged due to the lack of standardization and inferior performance of edema
Fig. 2 A patient with acute pericarditis. LGE imaging $(\mathbf{a}, \mathbf{c})$ shows pericardial enhancement as a bright line which encircles the heart. In Cine bSSFP imaging (b) pericardium looks like a black line and the fluid as a bright space with slightly higher signal intensity than the fat, pleural effusion is also shown in this image. In native T1 mapping (d), fat is black and can be easily differentiated from fluid which is bright

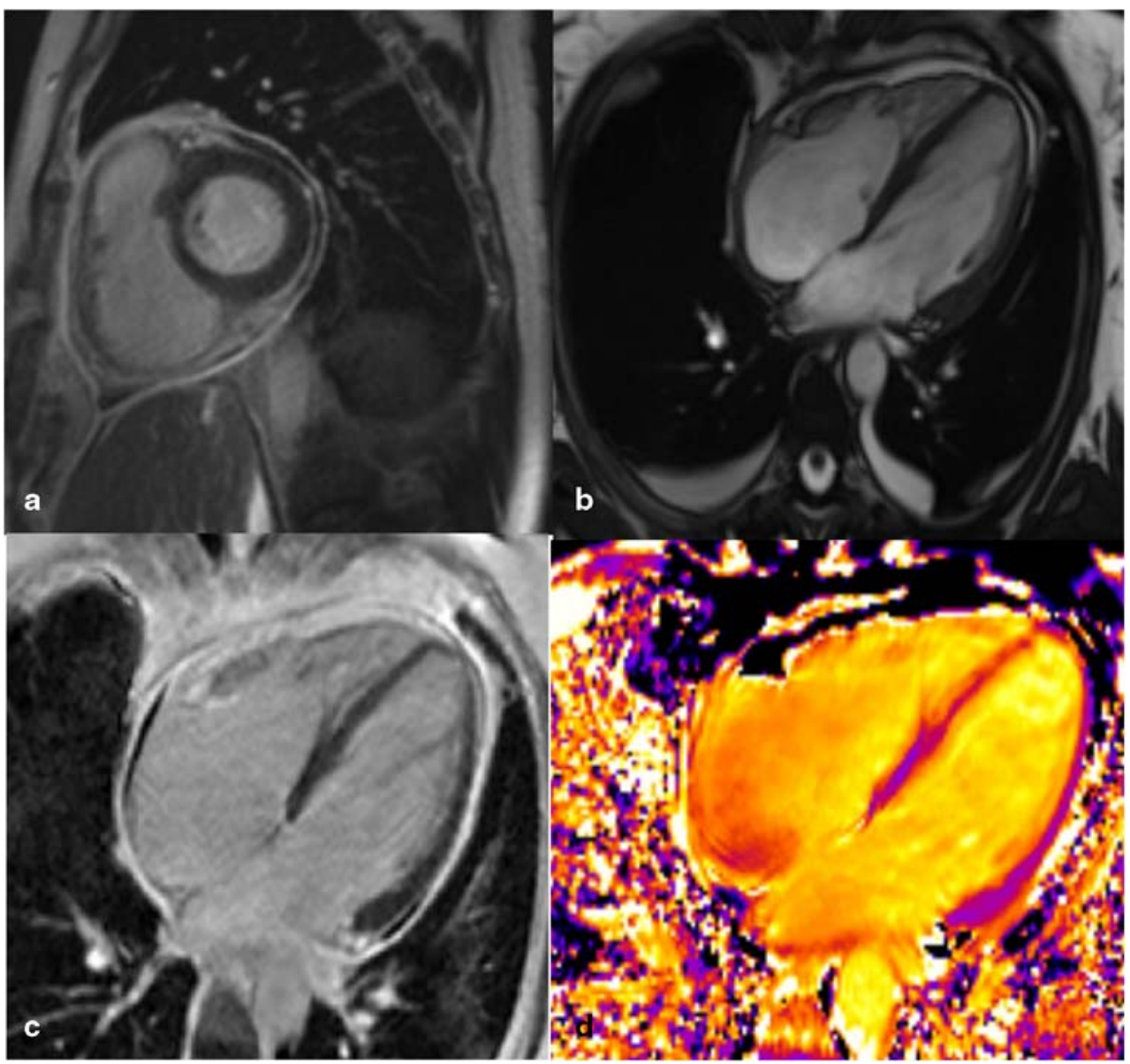


imaging (T2-weighted) as well as the complexity of normalizing T1 weighted images to peripheral muscle. The newest recommendations support a variety of techniques and their combinations for detecting inflamed myocardium. The highest accuracy has been reported from a combination of native T1 and T2 mapping. These techniques allow uncovering hidden myocardial injury and inflammation as well as determining the severity and acuity of the process, based on cut-off values, standardized image acquisition, and post-processing [9, 24].

\section{Pericardial Effusion, Tamponade and CMR}

Pericardial effusion may be accompanied with or without signs of pericardial inflammation. While most cases of pericardial effusion and pericarditis share the same causes, about $20 \%$ of cases remain unexplained (idiopathic). Prominent and persistent pericardial effusion is more frequent in malignancies and systemic diseases. A lot of patients with chronic kidney disease (CKD) demonstrate pericardial effusion as a part of cardiovascular involvement with accompanied myocardial remodeling that it is mainly detected on native T1 mapping technique [25]. Based on 2-dimensional echocardiography, the amount of pericardial effusion can be categorized as small $(<1 \mathrm{~cm})$, moderate $(1-2 \mathrm{~cm})$, large $(2-2,5 \mathrm{~cm})$, or very large ( $>$ $2,5 \mathrm{~cm})$ measured at end-diastole [26]. CMR criteria are based on the total amount of fluid, if the fluid on cine-SSFP or PSIRLGE imaging is circumferentially with a width of $<4 \mathrm{~mm}$ anterior to RV it is considered small, with $5 \mathrm{~mm}$ or more moderate (100-500 ml) (Fig. 3). Even though CMR is not the primary imaging modality for evaluation of pericardial effusion, it provides information on the location and the exact volume of the fluid [27]. By using the same volumetric techniques as used to calculate left ventricular volumes based on a full multi-slice cine SSFP image stack the amount of pericardial fluid can be determined. CMR can also identify clot and complex or loculated effusions. PSIR- LGE of the myo- or pericardium may demonstrate concurrent inflammation of these structures which contrasts with the low signal intensity of the pericardial fluid (black) in the same sequence. CMR tissue characterization may indicate loculated pericardial effusion or pericardial masses with or without signs of malignancy [28]. Due to the fact that transudates, exudates, hemorrhagic, and proteinaceous pericardial effusions, have different native $\mathrm{T} 1$ and $\mathrm{T} 2$ relaxation times, we are able to discriminate them by using mapping techniques [14]. It is demonstrated that T1 mapping of the pericardial fluid can be used to provide information about its composition. More specifically, a cut-off T1 value of $3013 \mathrm{~ms}$ can differentiate transudates from exudates with a sensitivity $94 \%$, specificity $79 \%$, and AUC of 0.86 (95\% CI 0.73-0.99), $p<0.0001$ [29] (Table 1). In addition native $\mathrm{T} 1$ and $\mathrm{T} 2$ mapping of the myocardium may reveal associated diffuse myocardial fibrosis or inflammation. Importantly, all these latter techniques are regarded as optional in the SCMR recommendations for standardized CMR due to the main focus on visualizing the amount of fluid and tissue characterization of the myocardium itself.

Cardiac tamponade is a life-threatening condition, which occurs when the cardiac chambers are compressed due to accumulation of fluid in the pericardial space. It is characterized by impaired cardiac filling and cardiac output. The amount of fluid which needs to accumulate in order to compress the cardiac chamber is related to the rate of its accumulation, as well as the elastic properties of the pericardium itself [30]. Given that a cardiac tamponade is an emergency situation, echocardiography is the preferred image modality for its diagnosis. Echocardiographic criteria of tamponade are; (a) presence of pericardial fluid, (b) dilated inferior vena cava, (c) reduced stroke volume, (d) collapse of the right ventricle in diastole, (e) right atrial collapse for more than one third of the cardiac cycle, and (f) respiratory variation of the mitral and tricuspid E velocity [31]. The use of CMR remains limited despite its ability to provide information when hemodynamic assessment with echocardiography is difficult and the diagnosis remains unclear [32]. CMR exam in case of tamponade must be a minimalistic scanning because anything beyond 5 min may not be tolerated by the patient or even be fatal. With cine SSFP imaging and real-time cine CMR imaging,
Fig. 3 A patient with circumferential pericardial effusion. LGE imaging (a) with little pericardial enhancement, fluid is depicted as a black space. Native T1 mapping (b), fluid has high native $\mathrm{T} 1$ values

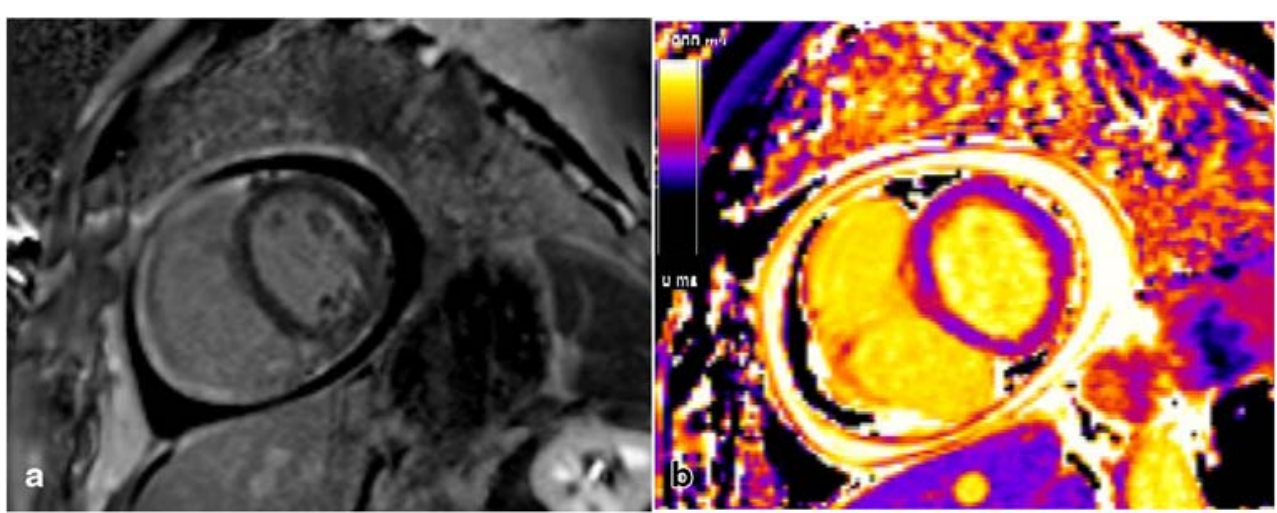


Table 1 A summary comparing different kinds of pericardial effusions according to the signal intensity (S.I) on T1 weighted imaging (T1W), T2 weighted imaging (T2W), and the T1 time in milliseconds (ms) in T1 mapping sequence

\begin{tabular}{llll}
\hline & T1W(S.I) & T2W(S.I) & T1 mapping \\
\hline Exudates & Medium & Medium & $>3013 \mathrm{~ms}$ \\
Transudates & Low & High & $<3013 \mathrm{~ms}$ \\
Hemorrhagic & High & High & - \\
Proteinaceous & High & Low & - \\
\hline
\end{tabular}

flattening of the interventricular septum, compression of the coronary sinus, distention of the superior vena cava, and the respiratory ventricular interdependence can be imaged.

\section{Constrictive Pericarditis and CMR}

Constrictive pericarditis (CP) occurs when the pericardium loses its normal elasticity and compliance and therefor compromises diastolic cardiac filling. All of the above may occur when the pericardium is subjected to chronic fibrous thickening and scarring as well as calcification [33]. Potential causes of constrictive pericarditis are; virus pericarditis, tuberculosis pericarditis, purulent pericarditis, cardiac surgery, radiotherapy, and idiopathic. Most cases of $\mathrm{CP}$ in developed countries are idiopathic and postsurgical $\mathrm{CP}$, whereas worldwide tuberculosis $\mathrm{CP}$ is the main presentation [34]. Constrictive pericarditis can be encountered in several forms such as; acute inflammatory (transient CP), effusive- constrictive pericarditis, and adhesive-constrictive pericarditis [35]. Acute inflammatory $\mathrm{CP}$ is commonly paired with acute pericarditis. Effusive $\mathrm{CP}$ and adhesive $\mathrm{CP}$ are more chronic processes [36]. In most cases, symptoms of $\mathrm{CP}$ are related to right heart failure and have a late onset requiring surgical pericardiectomy as the only therapeutic option. CMR can contribute to early detection and prompt treatment in order to avoid pericardiectomy or facilitate better surgical results.

Diagnosis of CP is challenging. Historically, cardiac catheterization has been the gold standard approach demonstrating equalization of diastolic pressures of the right and left ventricles, square root sign, rapid $\mathrm{x}$, and $\mathrm{y}$ descents of the atrial pressure curves and ventricular interdependence using the left ventricular and right ventricular pressures [37]. CMR is the only non-invasive image technique with a similar diagnostic accuracy as cardiac catheterization [38]. A thickened pericardium $>4 \mathrm{~mm}$ visualized with steady-state free precession (SSFP) imaging or LGE is indicative of constrictive pericarditis, when no active or ongoing inflammation is suspected $[39,40]$. Late gadolinium contrast enhancement of the pericardium as well as the high signal intensity on T2 weighted imaging or T1/T2 mapping are signs of ongoing inflammation and may lead to anti-inflammatory therapy rather than operation [6]. CMR may elucidate additional parameters of constriction such as the characteristic S- shaped intraventricular septal motion during the cardiac cycle (septal bounce) using SSFP, dilated inferior or superior vena cava or coronary sinus. Real time cine imaging may demonstrate the effect of free breathing on ventricular interdependence, a unique sign of constrictive pericarditis [41, 42]. Tagging has been proposed as an additional diagnostic criterion using the absence of slippage between the pericardium and the myocardium as a sign of CP [43]. More recently, real-time phage contrast CMR has been described to assess respiratory variations of $\mathrm{E}$ waves similar to echocardiography [44]. Again, only cine-imaging and LGE are regarded as mandatory in the SCMR recommendations for standardized CMR.

\section{Pericardial Masses and CMR}

Masses of the pericardium can be divided into primary masses $(0.001 \%$ to $0.03 \%$ incidence found in autopsies) or metastatic (1.7\% to $14 \%$ incidence found in autopsies). Metastatic tumors of the pericardium are the most common, with an estimated incidence 100 times more than the primary tumors [45]. Malignant processes of the pericardium can be presented as pericardial effusion (with or without tamponade), direct myocardial invasion, pericardial constriction, or pericarditis. Effusion (predominantly hemorrhagic) is being the most common [46]. Pericardial cysts are the most common benign pericardial lesions other benign tumors include fibroma, hemangioma, and lipoma. Pericardial mesothelioma is the most common primary malignant mass of the pericardium, accounting for approximately $50 \%$ of primary pericardial malignancies. Other primary malignancies include pericardial sarcoma (angiosarcoma more often) and primary pericardial lymphoma (usually diffuse large B cell type) (Fig. 4). Metastases to the pericardium are usually secondary to malignancies of the breast or lung, or melanoma. Thymic carcinoma, mediastinal teratoma or chest wall tumors are some rare tumors that can direct invade pericardium. Non-neoplastic pericardial masses include inflammatory pseudotumor (IgG4related disease) and tuberculosis pericarditis [47, 48].

Pericardial masses have a variant appearance on CMR according to their etiology. Primary tumors of the pericardium usually appear as a mass within the pericardial space. Metastatic disease is seen as discontinuation of the normal pericardium, thickening of the pericardium, or as pericardial effusion. Hematologic or lymphatic malignant spread, can be identified as, frequently multiple, focal nodular lesions or thickening of the pericardium, or as a pericardial effusion. Hemorrhagic or serosanguinous pericardial effusions have high signal intensity on T1 weighted sequences [45]. Apart from the anatomical information provided by CMR, it can also characterize the tissue composition of cystic lesions using "virtual histology" and is able 
Fig. 4 A young lady with lymphoma (red arrow) that invades from myocardium to pericardium. LGE imaging (a) and T1 mapping technique (b)

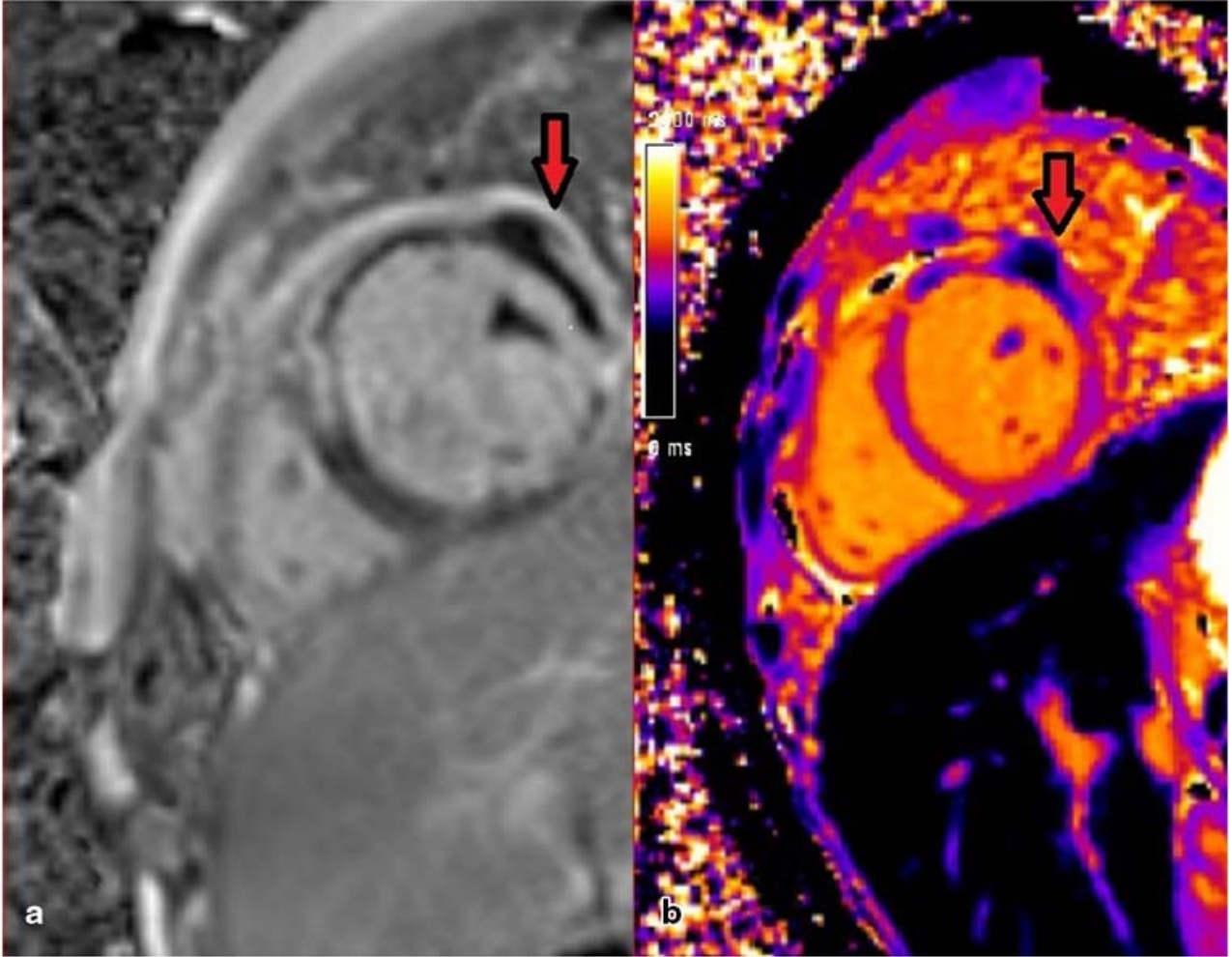

to assess the perfusion status of a pericardial mass. By using data from T1 weighted imaging and T2 weighted imaging as well as the late gadolinium enhancement some pericardial tumors can be defined with high confidence. Especially for pericardial lipomas, fat suppression technique help to identify a fat containing mass. In total, most of metastatic tumors have low signal intensity on T1 weighted imaging, high signal intensity on T2 weighted imaging and heterogeneous LGE. Melanoma is an exception to this rule as it demonstrates high signal intensity in T1 weighted imaging. Some novel techniques for differentiate the pericardial masses are dynamic contrast enhancement MRI as well as the mapping techniques. Native T1 mapping as well as T2 mapping can differentiate, with high accuracy, fat, thrombus, and fibrous containing masses [49] (Table 2). Of note, while CMR is strong in the detection of fat, the diagnosis on the exact tumor often remains unclear. CMR does provide important information on the malignant/benign origin of a mass and helps to define surgical approaches [50].

\section{Congenital Defect of the Pericardium and CMR}

Congenital absence of the pericardium is a rare disease with a prevalence from $0.007-0.015 \%$ in autopsy reports to $0.044 \%$ in surgical case. Congenital absence of the pericardium is classified into complete absence, complete right-sided absence, complete left-sided absence, partial right-sided absence, and partial left-sided absence. One of the most hazardous complication, is sudden cardiac death due to cardiac strangulation across the partial left-sided absence of the pericardium $[7,51]$.

CMR is useful to diagnose and classify congenital absence of the pericardium. While some quantitative parameters have been described based on small case series, e.g., whole-heart volume change (WHVC) more than $13 \%$, the main contribution of CMR is based on SFFP cine imaging visualizing atypical cardiac chamber movements and paradoxical septal motion due to the pericardial absence as well as the presence of lung tissue between the proximal segment of aorta and pulmonary artery $[52,53]$. CMR imaging may uncover some high-risk features that are potentially hazardous, like left ventricular myocardial crease or hinge point [7].

Table 2 A summary comparing different kinds of pericardial masses according to the signal intensity (S.I) in T1 weighted imaging (T1W), T2 weighted imaging ( $\mathrm{T} 2 \mathrm{~W})$, and the contrast media uptake in late gadolinium enhancement imaging (LGE)

\begin{tabular}{llll}
\hline & T1W (S.I) & T2W (S.I) & LGE (uptake) \\
\hline Cysts & Low & High & No \\
Lipomas & High & High & No \\
Lymphomas & Medium & Medium & No/minimal \\
Sarcomas & Medium & High & Heterogeneous \\
Metastatic & Low & High & Heterogeneous \\
Melanoma & High & High & Heterogeneous \\
\hline
\end{tabular}




\section{Treatment for Pericarditis and CMR}

The treatment of pericarditis requires monitoring of the therapy in order to achieve full recovery and minimum number of relapses. The emphasis lies on chronicity of pericarditis, which mandates a relatively long duration of treatment, and as such the use of safe drugs in long-term therapy. Although, Non-Steroidal Anti-Inflammatory Drugs (NSAIDs) and colchicine are proposed as the mainstay of therapy, we specifically favor the use of colchicine in low doses, whereas NSAIDs are best avoided due to nephrotoxic and cardiotoxic effects. It is worth noting that many patients with chronic perimyocarditis will also suffer from nephritis, as such NSAIDs are best avoided and never considered for longterm therapy, only used as per needed basis for acute pain. The cornerstone of pericarditis treatment is colchicine in low dose (usually $0.5 \mathrm{mg}$ colchicine once a day). It is worthwhile starting slowly with colchicine $0.5 \mathrm{mg}$ every other day, which largely avoids the gastrointestinal side effects. According to the patient's tolerance and the CMR follow up, colchicine therapy may be up-titrated to $0.5 \mathrm{mg}$ twice daily. Larger doses are usually not permissible as a long-term therapy, as they may cause severe diarrhea, nausea, cramping, abdominal pain, and vomiting. In auto-immune conditions, and specially in association with myocarditis, prednisone, and other diseasemodifying drugs may be considered [5].

CMR can help to monitor the response to therapy, identifying the successful elimination of pericarditis, as well as the persistent cases where longer duration of colchicine may be required [20]. According to the treatment response based on follow-up CMR results, the clinical course of pericarditis can be short with total remission of the disease in less than 4 months, or more resistant to the treatment with more persistent and longer clinical course. The disease is also likely to follow another pattern with remissions and exacerbations [10], $[12,54]$. Based on the fact that subclinical pericarditis and perimyocarditis is common in clinical practice, especially in autoimmune disease like Systemic Lupus Erythematosus (SLE) [55], it is more efficient to guide the treatment based on CMR results (Fig. 5).

CMR is also able to play a pivotal role in the management of pericarditis due to specific conditions. The most representative example is the autoimmune myopericarditis [56]. By using T1 and T2 mapping techniques as well as LGE an early diagnosis of the heart involvement in autoimmune disease such as SLE, Sarcoidosis or Rheumatoid Arthritis is possible [19]. CMR can detect early myopericardial involvement and suggest an up-titration of immunosuppressive therapy or adding colchicine to the existing therapeutic scheme. [24]. The treatment response as well as the relapse or reactivation of myopericardial autoimmune inflammation can be easily evaluated by using LGE imaging and the exact and comparable numbers of $\mathrm{T} 1$ and $\mathrm{T} 2$ mapping during patient's follow up, [18]. All in all CMR is able to be an important cardio-protector for patients with autoimmune diseases and mypericardial involvement as it can halt the ongoing inflammation of the heart

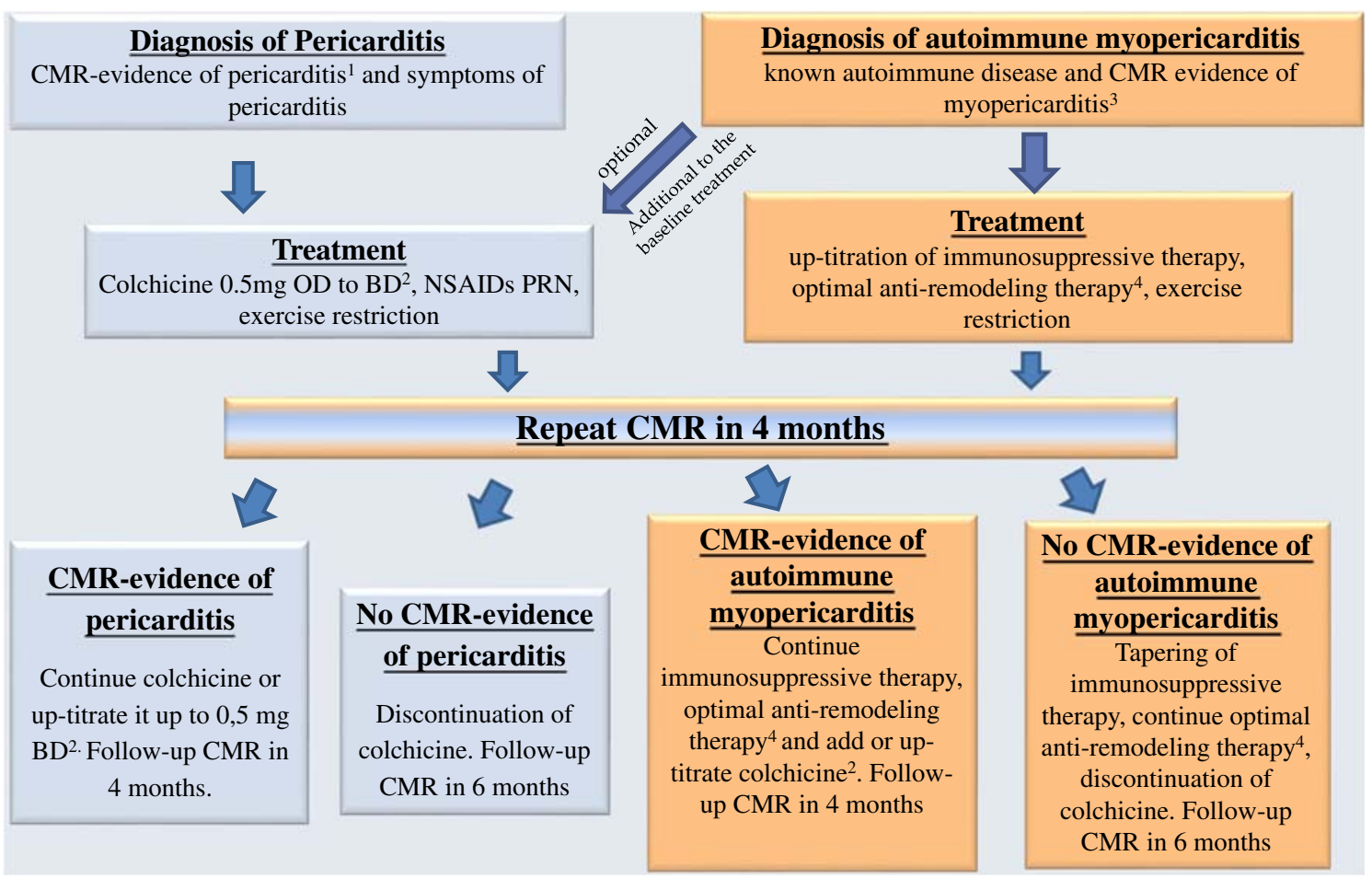

Fig. 5 Proposed therapeutic algorithm for pericarditis based on the CMR 
and the pericardium in a very early stage by guiding the immunosuppressive therapy $[24,57]$.

\section{Conclusion}

Pericardial diseases and disorders are relatively common in the everyday clinical practice with a multitude of clinical implications. Regularly, the first approach is made by echocardiography. On the other hand CMR, which is already used for some of these patients, is gaining ground in the pericardial diseases and frequently demonstrates pericardial abnormalities not detected by echocardiography. The classic CMR sequences are able to accurately depict the diseased and normal pericardium. Additional techniques, like tissue tagging of the pericardium, real time cine SSFP, native T1 and T2 mapping and real time phase contrast flow are able to boost our diagnostic capacity for all pericardial conditions. Especially the mapping techniques have replaced a variety of T1/T2 weighted imaging with and without fat suppression, as they provide a quantitative tissue analysis. Last but not the least, CMR provides a definitive diagnosis of pericarditis, constriction, myopericarditis, or pericardial effusion in less than 30 min with a single image modality, thus replacing other unnecessary imaging and diagnostic modalities such as laboratory tests, echocardiography, X-rays, CT, and catheterization.

Funding Information Open Access funding provided by Projekt DEAL. E.V was supported by training-research grant in memory of Maria Tsakos from the Hellenic Heart Foundation ELIKAR (www.elikar.gr) (research Fellowship, 2018-2019 to E.V).

E.N was supported by The German Centre for Cardiovascular Research (DZHK).

\section{Compliance with Ethical Standards}

Conflict of Interest All authors declare no conflict of interest.

Human and Animal Rights and Informed Consent This article does not contain any studies with human or animal subjects performed by any of the authors.

Open Access This article is licensed under a Creative Commons Attribution 4.0 International License, which permits use, sharing, adaptation, distribution and reproduction in any medium or format, as long as you give appropriate credit to the original author(s) and the source, provide a link to the Creative Commons licence, and indicate if changes were made. The images or other third party material in this article are included in the article's Creative Commons licence, unless indicated otherwise in a credit line to the material. If material is not included in the article's Creative Commons licence and your intended use is not permitted by statutory regulation or exceeds the permitted use, you will need to obtain permission directly from the copyright holder. To view a copy of this licence, visit http://creativecommons.org/licenses/by/4.0/.

\section{References}

1. Yusuf SW, Hassan SA, Mouhayar E, Negi SI, Banchs J, O'Gara PT. Pericardial disease: a clinical review. Expert Rev Cardiovasc Ther. 2016;14:525-39.

2. Mody P, Bikdeli B, Wang Y, Imazio M, Krumholz HM. Trends in acute pericarditis hospitalizations and outcomes among the elderly in the USA, 1999-2012. Eur Heart J Qual Care Clin Outcomes. 2018;4:98-105.

3. Cremer PC, Kumar A, Kontzias A, Tan CD, Rodriguez ER, Imazio $\mathrm{M}$, et al. Complicated pericarditis: understanding risk factors and pathophysiology to inform imaging and treatment. J Am Coll Cardiol. 2016;68:2311-28.

4. Puntmann VO, Valbuena S, Hinojar R, Petersen SE, Greenwood JP, Kramer CM, et al. Society for Cardiovascular Magnetic Resonance (SCMR) expert consensus for CMR imaging endpoints in clinical research: part I - analytical validation and clinical qualification. $\mathrm{J}$ Cardiovasc Magn Reson. 2018;20:67.

5. Adler Y, Charron P, Imazio M, Badano L, Barón-Esquivias G, Bogaert J, et al. 2015 ESC guidelines for the diagnosis and management of pericardial diseasesThe task force for the diagnosis and Management of Pericardial Diseases of the European Society of Cardiology (ESC)endorsed by: the European Association for Cardio-Thoracic Surgery (EACTS). Eur Heart J. 2015;36:2921-64.

6. Hinojar R, Botnar R, Kaski JC, Prasad S, Nagel E, Puntmann VO. Individualized cardiovascular risk assessment by cardiovascular magnetic resonance. Futur Cardiol. 2014;10:273-89.

7. Lopez D, Asher CR. Congenital absence of the pericardium. Prog Cardiovasc Dis. 2017;59:398-406.

8. Puntmann VO, Peker E, Chandrashekhar Y, Nagel E. T1 mapping in characterizing myocardial disease: a comprehensive review. Circ Res. 2016;119:277-99.

9. Puntmann VO, Zeiher AM, Nagel E. T1 and T2 mapping in myocarditis: seeing beyond the horizon of Lake Louise criteria and histopathology. Expert Rev Cardiovasc Ther. 2018;16:319-30.

10. Imazio M, Battaglia A, Gaido L, Gaita F. Recurrent pericarditis. Rev Med Interne. 2017;38:307-11.

11. Cummings KW, Green D, Johnson WR, Javidan-Nejad C, Bhalla S. Imaging of pericardial diseases. Semin Ultrasound CT MR. 2016;37:238-54.

12. Kumar A, Sato K, Yzeiraj E, Betancor J, Lin L, Tamarappoo BK, et al. Quantitative pericardial delayed Hyperenhancement informs clinical course in recurrent pericarditis. J Am Coll Cardiol Img. 2017;10:1337-46

13. D'Angelo T, Grigoratos C, Mazziotti S, Bratis K, Pathan F, Blandino A, et al. High-throughput gadobutrol-enhanced CMR: a time and dose optimization study. J Cardiovasc Magn Reson. 2017;19:83.

14. Al-Mallah MH, Almasoudi F, Ebid M, Ahmed AM, Jamiel A. Multimodality imaging of pericardial diseases. Curr Treat Options Cardiovasc Med. 2017;19:89.

15. Thavendiranathan P, Verhaert D, Walls MC, Bender JA, Rajagopalan S, Chung Y-C, et al. Simultaneous right and left heart real-time, free-breathing CMR flow quantification identifies constrictive physiology. JACC Cardiovasc Imaging. 2012;5:15-24.

16. Imazio M, Gaita F, LeWinter M. Evaluation and treatment of pericarditis: a systematic review. JAMA. 2015;314:1498-506.

17. Kobberøe SK, Körmendiné FD, Vera E, Krishnan B, Erik BH, Toft SH. Pericarditis as a Marker of Occult Cancer and a Prognostic Factor for Cancer Mortality. Circulation. 2017;136:996-1006.

18. Winau L, Hinojar Baydes R, Braner A, Drott U, Burkhardt H, Sangle S, et al. High-sensitive troponin is associated with subclinical imaging biosignature of inflammatory cardiovascular involvement in systemic lupus erythematosus. Ann Rheum Dis. 2018;77:1590-8. 
19. Hinojar R, Foote L, Sangle S, Marber M, Mayr M, Carr-White G, et al. Native T1 and T2 mapping by CMR in lupus myocarditis: disease recognition and response to treatment. Int $\mathrm{J}$ Cardiol. 2016;222:717-26

20. Alraies MC, AlJaroudi W, Yarmohammadi H, Yingchoncharoen T, Schuster A, Senapati A, et al. Usefulness of cardiac magnetic resonance-guided management in patients with recurrent pericarditis. Am J Cardiol. 2015;115:542-7.

21. $\mathrm{Xu} \mathrm{B}, \mathrm{K}$ won $\mathrm{DH}$, Klein AL. Imaging of the pericardium: a multimodality cardiovascular imaging update. Cardiol Clin. 2017:35:491-503.

22. Durazzo M, Gargiulo G, Pellicano R. Non-cardiac chest pain: a 2018 update. Minerva Cardioangiol. 2018;66:770-83.

23. Laufer-Perl M, Havakuk O, Shacham Y, Steinvil A, LetourneauShesaf S, Chorin E, et al. Sex-based differences in prevalence and clinical presentation among pericarditis and myopericarditis patients. Am J Emerg Med. 2017;35:201-5.

24. Puntmann VO, Isted A, Hinojar R, Foote L, Carr-White G, Nagel E. $\mathrm{T} 1$ and T2 mapping in recognition of early cardiac involvement in systemic Sarcoidosis. Radiology. 2017;285:63-72.

25. Chen M, Arcari L, Engel J, Freiwald T, Platschek S, Zhou H, et al. Aortic stiffness is independently associated with interstitial myocardial fibrosis by native $\mathrm{T} 1$ and accelerated in the presence of chronic kidney disease. Int J Cardiol Heart Vasc. 2019;24:100389.

26. Imazio M, Lazaros $\mathrm{G}$, Valenti $\mathrm{A}$, Carlini CCD, Maggiolini S, Pivetta E, et al. Outcomes of idiopathic chronic large pericardial effusion. Heart. 2019;105:477-81.

27. Halabi M, Faranesh AZ, Schenke WH, Wright VJ, Hansen MS, Saikus CE, et al. Real-time cardiovascular magnetic resonance subxiphoid pericardial access and pericardiocentesis using off-theshelf devices in swine. J Cardiovasc Magn Reson. 2013;15:61.

28. Azarbal A, LeWinter MM. Pericardial Effusion. Cardiol Clin. 2017:35:515-24.

29. Rosmini S, Seraphim A, Captur G, Gomes AC, Zemrak F, Treibel TA, et al. 247Characterisation of pleural and pericardial effusions with T1 mapping. European Heart Journal - Cardiovascular Imaging [Internet]. 2019 [cited 2019 Oct 30];20. Available from: https://doi.org/10.1093/ehjci/jez120.

30. Appleton C, Gillam L, Koulogiannis K. Cardiac Tamponade. Cardiol Clin. 2017;35:525-37.

31. Kishi P, Ahmad T, Dodd KW. Life-threatening development of cardiac Tamponade in the span of 24 hours. Clin Pract Cases Emerg Med. 2019;3:267-70.

32. Malik SB, Chen N, Parker RA, Hsu JY. Transthoracic echocardiography: pitfalls and limitations as delineated at cardiac CT and MR imaging. Radiographics. 2017;37:383-406.

33. Welch TD, Oh JK. Constrictive pericarditis. Cardiol Clin. 2017;35: 539-49.

34. Porta-Sánchez A, Sagristà-Sauleda J, Ferreira-González I, TorrentsFernández A, Roca-Luque I, García-Dorado D. Constrictive Pericarditis: Etiologic Spectrum, Patterns of Clinical Presentation, Prognostic Factors, and Long-term Follow-up. Rev Esp Cardiol (Engl Ed). 2015;68:1092-100.

35. Miranda WR, Oh JK. Constrictive pericarditis: a practical clinical approach. Prog Cardiovasc Dis. 2017;59:369-79.

36. Miranda WR, Oh JK. Effusive-constrictive pericarditis. Cardiol Clin. 2017;35:551-8.

37. Geske JB, Anavekar NS, Nishimura RA, Oh JK, Gersh BJ. Differentiation of constriction and restriction: complex cardiovascular hemodynamics. J Am Coll Cardiol. 2016;68:2329-47.

38. Aquaro GD, Barison A, Cagnolo A, Todiere G, Lombardi M, Emdin M. Role of tissue characterization by cardiac magnetic resonance in the diagnosis of constrictive pericarditis. Int J Cardiovasc Imaging. 2015;31:1021-31.
39. Kumawat M, Lahiri TK, Agarwal D. Constrictive pericarditis: retrospective study of 109 patients. Asian Cardiovasc Thorac Ann. 2018;26:347-52.

40. Young PM, Glockner JF, Williamson EE, Morris MF, Araoz PA, Julsrud PR, et al. MR imaging findings in 76 consecutive surgically proven cases of pericardial disease with $\mathrm{CT}$ and pathologic correlation. Int J Cardiovasc Imaging. 2012;28:1099-109.

41. Vermersch M, Longère B, Coisne A, Schmidt M, Forman C, Monnet A, et al. Compressed sensing real-time cine imaging for assessment of ventricular function, volumes and mass in clinical practice. Eur Radiol. 2019.

42. Sun A, Zhao B, Li Y, He Q, Li R, Yuan C. Real-time phase-contrast flow cardiovascular magnetic resonance with low-rank modeling and parallel imaging. J Cardiovasc Magn Reson. 2017;19:19.

43. Glower DD. Sticking points in magnetic resonance diagnosis of constrictive pericarditis. J Thorac Cardiovasc Surg. 2016;151:1356-7.

44. Anavekar NS, Wong BF, Foley TA, Bishu K, Kolipaka A, Koo CW, et al. Index of biventricular interdependence calculated using cardiac MRI: a proof of concept study in patients with and without constrictive pericarditis. Int J Cardiovasc Imaging. 2013;29:363-9.

45. Tower-Rader A, Kwon D. Pericardial masses, cysts and diverticula: a comprehensive review using multimodality imaging. Prog Cardiovasc Dis. 2017;59:389-97.

46. Maleszewski JJ, Anavekar NS. Neoplastic pericardial disease. Cardiol Clin. 2017;35:589-600.

47. Raeside MC, Gormly K, Neuhaus SJ, Kotasek D, James C. Primary pericardial mesothelioma presenting as multiple pericardial masses on CT. BJR Case Rep. 2016;2:20150295.

48. Yassi U, Iqbal F, Stevenson HL. IgG4-related sclerosing pericarditis in a young male with recurrent chest pain. Ann Thorac Surg. 2019.

49. Caspar T, El Ghannudi S, Ohana M, Labani A, Lawson A, Ohlmann P, et al. Magnetic resonance evaluation of cardiac thrombi and masses by T1 and T2 mapping: an observational study. Int J Cardiovasc Imaging. 2017;33:551-9.

50. Haslbauer JD, Lindner S, Valbuena-Lopez S, Zainal H, Zhou H, D'Angelo T, et al. CMR imaging biosignature of cardiac involvement due to cancer-related treatment by $\mathrm{T} 1$ and $\mathrm{T} 2$ mapping. Int $\mathrm{J}$ Cardiol. 2019;275:179-86.

51. Xu B, Betancor J, Asher C, Rosario A, Klein A. Congenital absence of the pericardium: a systematic approach to diagnosis and management. Cardiology. 2017;136:270-8.

52. Palau P, Domínguez E, García-González P, Gallego J, Bosch MJ, Sieso E. Isolated Partial Congenital Absence of the Pericardium: A Familial Presentation. Can J Cardiol. 2016:32-1039.e1-2.

53. Macaione F, Barison A, Pescetelli I, Pali F, Pizzino F, Terrizzi A, et al. Quantitative criteria for the diagnosis of the congenital absence of pericardium by cardiac magnetic resonance. Eur J Radiol. 2016;85:616-24.

54. Imazio M, Battaglia A, Gaido L, Gaita F. Recurrent pericarditis. Rev Med Interne. 2017;38:307-11.

55. Puntmann VO, D'Cruz D, Smith Z, Pastor A, Choong P, Voigt T, et al. Native myocardial T1 mapping by cardiovascular magnetic resonance imaging in subclinical cardiomyopathy in patients with systemic lupus erythematosus. Circ Cardiovasc Imaging. 2013;6:295-301.

56. Mavrogeni S, Koutsogeorgopoulou L, Dimitroulas T, MarkousisMavrogenis G, Kolovou G. Complementary role of cardiovascular imaging and laboratory indices in early detection of cardiovascular disease in systemic lupus erythematosus. Lupus. 2017;26:227-36.

57. Mavrogeni S, Markousis-Mavrogenis G, Koutsogeorgopoulou L, Kolovou G. Cardiovascular magnetic resonance imaging: clinical implications in the evaluation of connective tissue diseases. J Inflamm Res. 2017;10:55-61.

Publisher's Note Springer Nature remains neutral with regard to jurisdictional claims in published maps and institutional affiliations. 